Supplement of Solid Earth, 10, 329-342, 2019 https://doi.org/10.5194/se-10-329-2019-supplement (c) Author(s) 2019. This work is distributed under the Creative Commons Attribution 4.0 License.

(c) (i)

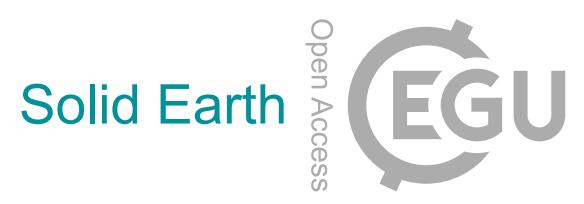

Supplement of

\title{
Precision of continuous GPS velocities from statistical analysis of synthetic time series
}

Christine Masson et al.

Correspondence to: Christine Masson (christine.masson@umontpellier.fr)

The copyright of individual parts of the supplement might differ from the CC BY 4.0 License. 


\section{Section S1 Effect of resolved offsets}

This figure shows the regression tree for Section 3.2.2 about the effect of several resolved offset.

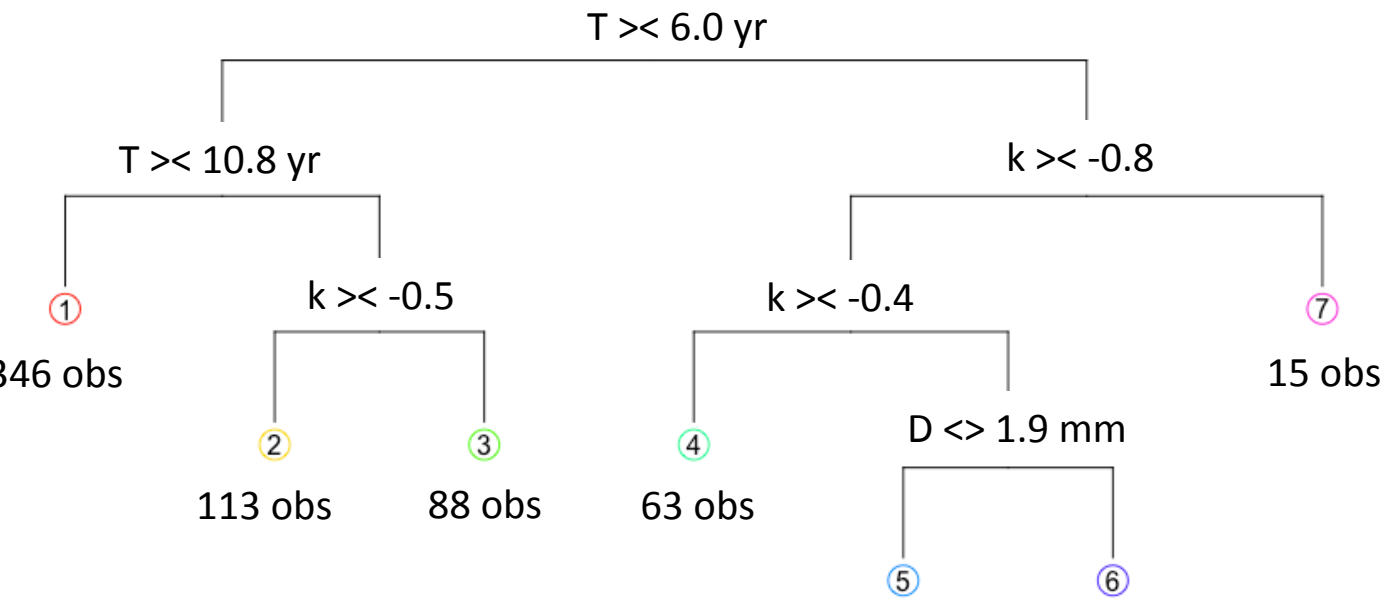

34 obs 69 obs

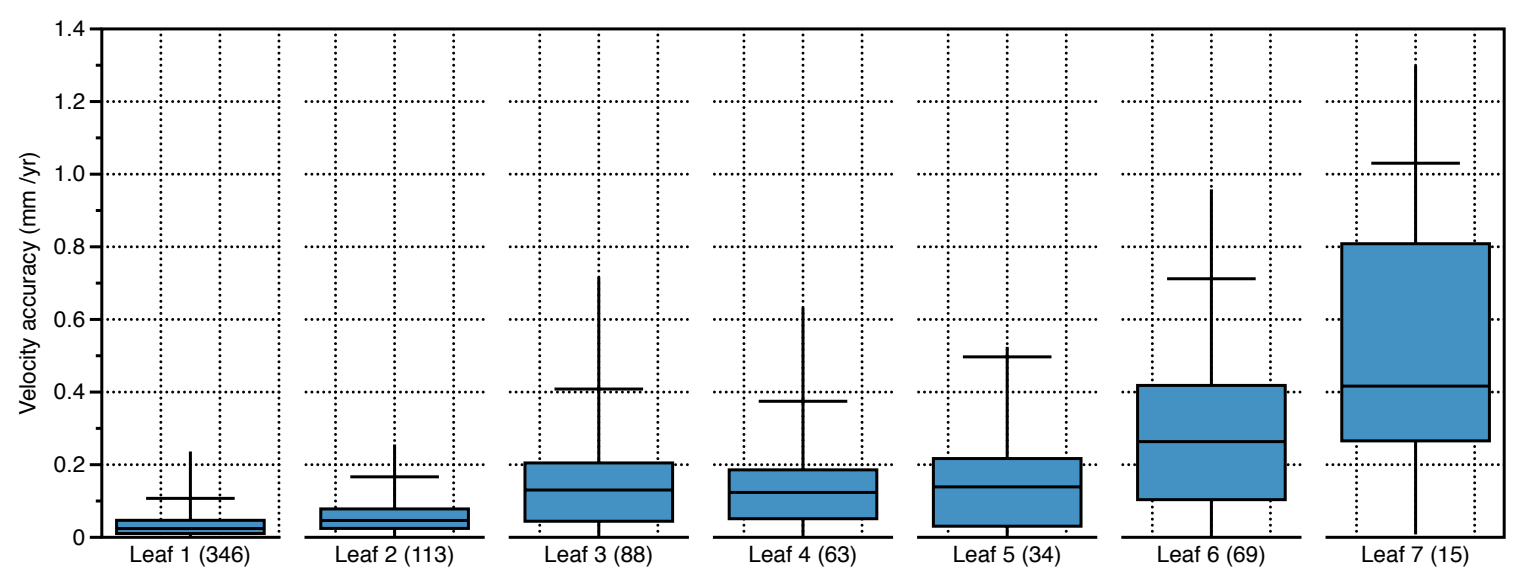

5 Figure S1: The tree classification (a) and the whisker plots of the associated leaves (b) for the full dataset with multiple resolved offsets, variable noise and seasonal signals.

\section{Section S2 Methodology of offset detection}

In order to choose the optimum values of the parameters $b$ (significance threshold) and $\Delta d$ (offset separation date), we used a subset of data (450 series) representative of the characteristics of section 2 . We tested 9 combinations of parameters with $b=$ $15,20,25$ and $\Delta d=10,20,35$. Then we compared the percentage of offsets detected, relative to the total number of offsets, 
as well as the number of false detections (i.e., no actual offset), relative to the total number of detections (Fig B). A first remark is related to the calculation time required for the $\Delta d=10$ analyzes, which is 10 times higher than for $\Delta d=20$. In addition, the results obtained are only slightly better for false detections but lower for the percentage of true detections. Analyzes with $b=25$ or $\Delta d=35$ clearly give poorer results. The results of the combinations $b=15-\Delta d=20$ and $b=20-\Delta d$ $5=20$ show very similar results (difference $<1 \%$ ), we finally chose the combination $b=20-\Delta d=20$. For reference, partial tests with $b=10$ showed a dramatic increase of false detections, so we decided not to apply it to the entire dataset.

Regarding the false detections, we have several indicators allowing us to discern part of them, considering that they are commonly associated with a very large uncertainty and are easily identifiable, but this is not the case for all. They are mainly related to the noise structure (small k), which can create several false detections very close together. There remains a part of false detections not discernible.

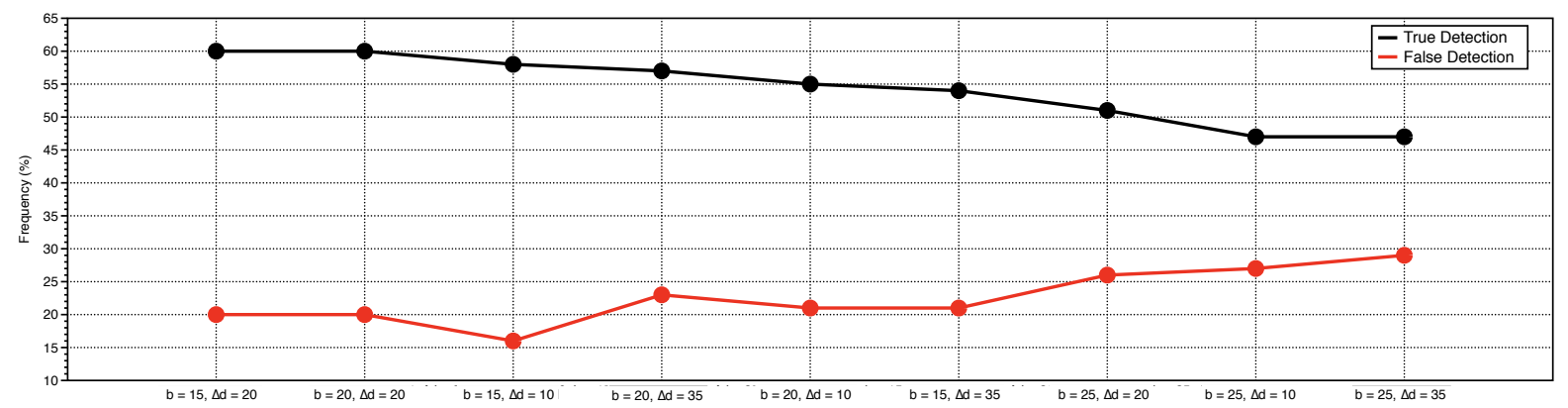

Figure S2: Percentages of true and false detections based on combinations of $b$ and $\Delta d$. 